To appear in the International Journal of General Systems

Vol. 00, No. 00, May 2014, 1-14

\title{
Receding Horizon Filtering for a Class of Discrete Time-Varying Nonlinear Systems with Multiple Missing Measurements
}

\author{
Derui Ding ${ }^{a *}$ Zidong Wang ${ }^{b, c}$ Fuad E. Alsaadi $^{c}$ and Bo Shen ${ }^{a}$ \\ ${ }^{a}$ School of Information Science and Technology, Donghua University, \\ Shanghai 200051, China \\ ${ }^{b}$ Department of Computer Science, Brunel University, \\ Uxbridge, Middlesex UB8 3PH, United Kingdom \\ ${ }^{c}$ Communication Systems and Networks (CSN) Research Group, Faculty of Engineering, \\ King Abdulaziz University, Jeddah 21589, Saudi Arabia \\ (Received 00 Month 201X; final version received 00 Month 201X)
}

\begin{abstract}
This paper is concerned with the receding horizon filtering problem for a class of discrete time-varying nonlinear systems with multiple missing measurements. The phenomenon of missing measurements occurs in a random way and the missing probability is governed by a set of stochastic variables obeying the given Bernoulli distribution. By exploiting the projection theory combined with stochastic analysis techniques, a Kalman-type receding horizon filter is put forward to facilitate the online applications. Furthermore, by utilizing the conditional expectation, a novel estimation scheme of state covariance matrices is proposed to guarantee the implementation of the filtering algorithm. Finally, a simulation example is provided to illustrate the effectiveness of the established filtering scheme.
\end{abstract}

Keywords: Receding horizon filtering; discrete time-varying systems; stochastic nonlinear; multiple missing measurements

\section{Introduction}

In signal processing and control areas, the Kalman filter (KF) has been regarded as an efficiently recursive filter to estimate unknown system states by utilizing measurements. However, since undesirable signals with internal states may accumulate, the traditional KF algorithms might suffer from persistent modeling errors (Fitzgerald, 1971; Sangsuk \& Bullock, 1990) or numerical errors (Grewal \& Anderews, 1993). As such, it could take a long time to converge to a real state even though the temporary undesirable errors disappear. To improve its performance, an alternative filter, namely, the receding horizon filter, has been proposed in the past few years. The main idea of this kind of filter is to estimate the state vector in terms of finite measurement samples, and then discard the oldest measurement sample as a new sample becomes available. It is not difficult to see that the memory length of the filter is fixed. As discussed by Kwon et al. (1994), the receding horizon filter owns a series of good numerical characteristics such as the bounded input bounded output (BIBO) stability, the low round-off noise, the no limit cycles compared to the infinite impulse response(IIR), and so forth. Therefore, such

*Corresponding author. Email: deruiding2010@gmail.com 
a filtering approach has been widely applied in various engineering problems, for instance, signal processing, control system design, mobile target tracking, wireless positioning system, see Alessandri et al. (2008, 2003); Buxbaum et al. (1974); Friedlander et al. (1978); Jazwinski (1968); Kim (2010); Kwon et al. (1989, 1994, 2002); Park et al. (1997); Shmaliy et al. (2008); Shmaliy (2008) and the references therein.

As the ever-increasing popularity of communication networks, the limited bandwidth of the communication channel inevitably leads to some network induced phenomena which have gained much research effort in the past decade (Hounkpevi \& Yaz, 2007; Kluge et al., 2010; Liang et al., 2014; Liu et al., 2014; Sahebsara et al., 2007b; Sinopoli et al., 2004; Wang et al., 2003). Recently, the so-called multiple missing measurement has been considered to account for the case that each sensor owns a different occurrence probability of missing measurements in an array of sensors, see (Bouibed et al., 2014; Dong et al., 2010; Hu et al., 2012; Shen et al., 2010; Shen, 2014; Wei et al., 2009) for more details. On the other hand, nonlinearities exist universally in practice that have long been one of the main research streams. In a networked environment, the nonlinearities might stem from the random fluctuation of the network load and the unreliability of the wireless links and therefore such nonlinearities themselves could experience random abrupt changes in their type or intensity, see e.g. (He et al., 2013; Hu et al., 2012, 2013; Jacobson, 1974; Wei \& Yaz, 2001). As such, it seems more reasonable to design the receding horizon filter by taking both the multiple missing measurements and the stochastic nonlinearities into account.

To the best of the authors' knowledge, the receding horizon filtering problem for discrete time-varying systems with multiple missing measurements or stochastic nonlinearities has not been adequately investigated, despite its clear engineering insight. The main reason is that the introduced phenomena would pose significant challenges on the design of receding horizon filters. For instance, in this case, the mean and covariance of the initial state would be unknown in the sliding-window because a priori information is hardly available. Furthermore, traditional methods handling covariance matrices of filtering error, such as setting values (e.g. $\infty I$ ), least-squares techniques or maximum-likelihood methods, are no longer valid due primarily to the coupling between the filtering error and the system state. As such, the purpose of this paper is to shorten such a gap.

In this paper, we aim to examine the impact of missing measurements and stochastic nonlinearities on the design of receding horizon filters. By employing the projection theory combined with the stochastic analysis techniques, a receding horizon Kalman-type filter is designed and a novel estimation method is provided to obtain the covariance matrix of the filtering error. Moreover, a simulation example is proposed to show the effectiveness of the established receding horizon filtering scheme. The main contributions of this paper are outlined as follows: 1) the system under consideration is comprehensive to cover several network-induced phenomena; 2) a kind of receding horizon filter is derived for the purpose of online applications; and 3) a novel estimation scheme for the state covariance matrix is provided by employing the augmented method in order to guarantee the implementation of the filtering algorithm.

The rest of this paper is organized as follows. In Section 2, a class of discrete time-varying nonlinear systems with multiple missing measurements and stochastic nonlinearities are introduced. In Section 3, by using the projection theory, some recursive algorithms of receding horizon filtering are derived, and then the estimation of filtering error covariance matrix is carried out in terms of the conditional expectation. Furthermore, a simulation example is given in Section 4 to demonstrate 
the effectiveness. Finally, we conclude the paper in Section 5.

Notation The notation used here is fairly standard except where otherwise stated. $\mathrm{R}^{n}$ and $\mathrm{R}^{n \times m}$ denote, respectively, the $n$-dimensional Euclidean space and the set of all $n \times m$ real matrices. $I$ denotes the identity matrix of compatible dimension. The notation $X \geq Y$ (respectively, $X>Y$ ), where $X$ and $Y$ are symmetric matrices, means that $X-Y$ is positive semi-definite (respectively, positive definite). $A^{T}$ and $r(A)$ represent the transpose and rank of the matrix $A$, respectively. Moreover, we may fix a probability space $(\Omega, \mathscr{F}$, Prob), where Prob, the probability measure, has total mass 1 . $\mathrm{E}\{x\}$ stands for the expectation of the stochastic variable $x$ with respect to the given probability measure Prob. $\operatorname{diag}\{\cdots\}$ stands for a block-diagonal matrix. The symbol $\otimes$ and $\circ$ denote the Kronecker product and the Hadamard product, respectively.

\section{Main strategies of distributed filtering}

Consider the following discrete time-varying nonlinear system:

$$
\left\{\begin{aligned}
x_{k+1} & =A_{k} x_{k}+f\left(x_{k}, \vartheta_{k}\right)+D_{k} w_{k} \\
y_{k} & =\Xi_{k} C_{k} x_{k}+E_{k} v_{k}
\end{aligned}\right.
$$

where $x_{k} \in \mathrm{R}^{n_{x}}$ represents the state vector, $y_{k} \in \mathrm{R}^{n_{y}}$ is the measurement output, and $w_{k} \in \mathrm{R}^{p}, v_{k} \in \mathrm{R}^{q}$ and $\vartheta_{k} \in \mathrm{R}^{s}$ are mutually independent zero-mean white Gaussian sequences with covariances $Q, R$ and $I$, respectively. $A_{k}, C_{k}, D_{k}$ and $E_{k}$ are known matrices with appropriate dimensions. $\Xi_{k}=\operatorname{diag}\left\{\alpha_{1, k}, \alpha_{2, k}, \cdots, \alpha_{n_{y}, k}\right\}$ with $\alpha_{i, k}\left(i=1,2, \cdots, n_{y}\right)$ being $n_{y}$ unrelated random variables, which are also unrelated with $w_{k}, v_{k}, \vartheta_{k}$ and $x_{0}$. Furthermore, the stochastic variable $\alpha_{i, k}$ is assumed to be a Bernoulli-distributed white sequence taking values on 0 or 1 with:

$$
\operatorname{Prob}\left\{\alpha_{i, k}=0\right\}=1-\bar{\alpha}_{i}, \quad \operatorname{Prob}\left\{\alpha_{i, k}=1\right\}=\bar{\alpha}_{i} .
$$

The function $f\left(x_{k}, \vartheta_{k}\right)$ with $f\left(0, \vartheta_{k}\right)=0$ is a stochastic nonlinearity (Wei et al., 2009) having the following first moment for all $x_{k}$ :

$$
\mathrm{E}\left\{f\left(x_{k}, \vartheta_{k}\right) \mid x_{k}\right\}=0
$$

and the covariances given by

$$
\mathrm{E}\left\{f\left(x_{k}, \vartheta_{k}\right) f^{T}\left(x_{i}, \vartheta_{i}\right) \mid x_{k}\right\}=0, \quad i \neq k,
$$

and

$$
\mathrm{E}\left\{f\left(x_{k}, \vartheta_{k}\right) f^{T}\left(x_{k}, \vartheta_{k}\right) \mid x_{k}\right\}=\sum_{i=1}^{m} \Pi_{i} x_{k}^{T} \Gamma_{i} x_{k},
$$

where $m$ is a known integer, $\Pi_{i}$ and $\Gamma_{i}$ are known matrices with appropriate dimensions.

As discussed in Introduction, this paper focuses on the receding horizon filtering problem, where the length of sliding-window is $N+1$. Generally speaking, the receding horizon filter employs only the finite measurements on the interval $[k-$ $N, k]$ and discards the oldest measurements when a new measurement is available. Denoted the information set as $\mathcal{I}_{k}^{N}=\left\{y_{k_{N}}, y_{k_{N}+1}, \cdots, y_{k}\right\}$ with $k_{N}=k-N$, the 
filter is described by

$$
\hat{x}_{(k \mid k ; N)}=\sum_{i=k_{N}}^{k} H_{[k, i ; N]} y_{i}, \quad y_{i} \in \mathcal{I}_{k}^{N}
$$

where $\hat{x}_{(k \mid k ; N)}$ denotes the state estimate of system (1) at time $k$ and $H_{[k, i ; N]}$ is the filter parameter to be designed.

Our aim in this paper is to design a filter with the form (5) for system (1) with stochastic nonlinearities and missing measurements. In other words, we are going to determine the filter parameters $H_{[k, i ; N]}\left(k_{N} \leq i \leq k\right)$ such that the following orthogonality is satisfied:

$$
\mathrm{E}\left\{\left(x_{k}-\hat{x}_{(k \mid k ; N)}\right) y_{i}^{T}\right\}=0, \quad k_{N} \leq i \leq k
$$

\section{Main results}

In this section, we will provide a recursive algorithm of the receding horizon filter and then derive an estimation of state covariance matrix for the discrete timevarying nonlinear systems (1) to apply the established recursive algorithm.

\subsection{The design of receding horizon filter}

Before proceeding further, we introduce the following lemma which provides a fundamental iteration algorithm to design the filter parameter $H_{[k, i ; N]}$ by handling a set of Riccati-type difference equations. Note that this lemma can be showed along the similar lines of the proofs of Theorem 2 in Kwon et al. (1989) and Theorem 2.1 in Kwon et al. (1994), and therefore the proof is omitted to the space saving.

Lemma 1. Let the state covariance matrix $\mathrm{E}\left\{x_{k_{N}-1} x_{k_{N}-1}^{T} \mid \mathcal{I}_{k}^{N}\right\}:=\mathcal{P}_{\left[k_{N}-1, k_{N}-1 ; N\right]}$ be given. For the discrete time-varying nonlinear system (1) with multiple missing measurements, the filter parameters are uniquely determined by the following iteration algorithms

1) $H_{[k, i ; N]}=\left.H_{[k, i ; n]}\right|_{n=N}$ for the fixed $k$ and $N$, where $H_{[k, i ; n]}$ satisfies the recursive matrix difference equations

$$
\left\{\begin{aligned}
& H_{\left[k, i ; i-k_{N}\right]}=\mathcal{R}_{\left[k, i-k_{N} ; N\right]} C_{i}^{T} \Xi \mathcal{S}_{[i ; N]}^{-1}, \\
& H_{[k, i ; n+1]}=\left(I-\mathcal{R}_{[k, n+1 ; N]} C_{k_{N}+n+1}^{T} \Xi \mathcal{S}_{\left[k_{N}+n+1 ; N\right]}^{-1} \Xi C_{k_{N}+n+1}\right) A_{k_{N}+n} H_{[k, i ; n]} \\
& k_{N} \leq i \leq k, \quad i-k_{N} \leq n \leq N-1 ;
\end{aligned}\right.
$$

or 2) $H_{[k, i ; N]}$ calculated directly by

$$
\left\{\begin{array}{lr}
H_{[k, i ; N]}=\Phi_{\left[N, i-k_{N}\right]}^{k} \mathcal{R}_{\left[k, i-k_{N} ; N\right]} C_{i}^{T} \Xi \mathcal{S}_{[i ; N]}^{-1}, & k_{N} \leq i \leq k, \\
\Phi_{[N, m]}^{k}=\Phi_{[N, m+1]}^{k}\left(I-\mathcal{R}_{[k, m+1 ; N]} C_{k_{N}+m+1}^{T} \Xi \mathcal{S}_{\left[k_{N}+m+1 ; N\right]}^{-1} \Xi C_{k_{N}+m+1}\right) A_{k_{N}+m} \\
\Phi_{[N, N]}^{k}=I, & 0 \leq m \leq N-1,
\end{array}\right.
$$


where

$$
\begin{aligned}
& \mathcal{R}_{[k, s+1 ; N]}=\overline{\mathcal{R}}_{[k, s ; N]}-\overline{\mathcal{R}}_{[k, s ; N]} C_{k_{N}+s+1}^{T} \Xi \tilde{\mathcal{S}}_{\left[k_{N}+s+1 ; N\right]}^{-1} \Xi C_{k_{N}+s+1} \overline{\mathcal{R}}_{[k, s ; N]}, \\
& \overline{\mathcal{R}}_{[k, s ; N]}=A_{k_{N}+s} \mathcal{R}_{[k, s ; N]} A_{k_{N}+s}^{T}+\sum_{l=1}^{m} \Pi_{l} \operatorname{tr}\left(\mathcal{P}_{\left[k_{N}+s, k_{N}+s ; N\right]} \Gamma_{l}\right)+D_{k_{N}+s} Q D_{k_{N}+s}^{T}, \\
& \tilde{\mathcal{S}}_{\left[k_{N}+n+1 ; N\right]}=\mathcal{S}_{\left[k_{N}+n+1 ; N\right]}+\Xi C_{k_{N}+n+1} \overline{\mathcal{R}}_{[k, n ; N]} C_{k_{N}+n+1}^{T} \Xi, \quad s \geq-1, \\
& \mathcal{P}_{[r+1, r+1 ; N]}=A_{r} \mathcal{P}_{[r, r ; N]} A_{r}^{T}+\sum_{l=1}^{m} \Pi_{l} \operatorname{tr}\left(\mathcal{P}_{[r, r ; N]} \Gamma_{l}\right)+D_{r} Q D_{r}^{T}, \quad r \geq k_{N}-1, \\
& \mathcal{R}_{[k,-1 ; N]}=\mathcal{P}_{\left[k_{N}-1, k_{N}-1 ; N\right]}, \quad \mathcal{S}_{[r ; N]}=\tilde{\Xi} \circ\left(C_{r} \mathcal{P}_{[r, r ; N]} C_{r}^{T}\right)+E_{r} R E_{r}^{T}, \\
& \Xi=\operatorname{diag}\left\{\bar{\alpha}_{1}, \bar{\alpha}_{2}, \cdots, \bar{\alpha}_{n_{y}}\right\}, \quad \tilde{\Xi}=\operatorname{diag}\left\{\bar{\alpha}_{1}\left(1-\bar{\alpha}_{1}\right), \bar{\alpha}_{2}\left(1-\bar{\alpha}_{2}\right), \cdots, \bar{\alpha}_{n_{y}}\left(1-\bar{\alpha}_{n_{y}}\right)\right\} .
\end{aligned}
$$

In the above lemma, two algorithms of the receding horizon filter have been provided. Now, it is interesting to investigate how to modify the filter parameters by using the obtained parameters when the length of sliding-windows is increased, and how to design a Kalman-type receding horizon filter for the easy implementation. As such, in terms of the information sets $\mathcal{I}_{k+1}^{N+1}$ and $\mathcal{I}_{k}^{N}$, the relationship on the designed filter parameters is revealed in the following theorem.

Theorem 1. Let $\mathcal{P}_{\left[k_{N}-1, k_{N}-1 ; N\right]}$ and $\mathcal{P}_{\left[k_{N}-1, k_{N}-1 ; N+1\right]}$ be given. For the discrete time-varying nonlinear systems (1) with multiple missing measurements, there exists the following Kalman-type receding horizon filter

$$
\hat{x}_{(k+1 \mid k+1 ; N+1)}=A_{k} \hat{x}_{(k \mid k ; N)}+K_{k+1}\left(y_{k+1}-\Xi C_{k+1} A_{k} \hat{x}_{(k \mid k ; N)}\right)
$$

with the filter gain

$$
K_{k+1}=\left(\overline{\mathcal{R}}_{[k, N ; N]}+\Delta_{k+1}^{\mathcal{H}}\right) C_{k+1}^{T} \Xi \Upsilon_{k+1}^{-1}
$$

and the filtering error covariance

$$
\mathcal{R}_{[k+1, N+1 ; N+1]}=\frac{1}{2}\left\{\left(I-K_{k+1} \Xi C_{k+1}\right) \Delta_{\mathcal{R}}^{\mathcal{H}}+\left(\Delta_{\mathcal{R}}^{\mathcal{H}}\right)^{T}\left(I-K_{k+1} \Xi C_{k+1}\right)^{T}\right\}
$$

where

$$
\begin{aligned}
& \mathcal{A}_{(k: i)}:=A_{k} A_{k-1} \cdots A_{i}(\forall k>i), \quad \mathcal{A}_{(i-1: i)}:=I, \\
& \Delta_{[s, k+1]}^{\mathcal{P}}=\mathcal{P}_{[s, k+1 ; N+1]}-\mathcal{P}_{[s, k+1 ; N]}=\left(\mathcal{P}_{[s, s ; N+1]}-\mathcal{P}_{[s, s ; N]}\right) \mathcal{A}_{(k: s)}^{T}, \\
& \Delta_{k+1}^{\mathcal{H}}=\Delta_{[k+1, k+1]}^{\mathcal{P}}-A_{k} \sum_{s=k_{N}}^{k} H_{[k, s ; N]} \Xi C_{s} \Delta_{[s, k+1]}^{\mathcal{P}}, \\
& \Delta_{\mathcal{R}}^{\mathcal{H}}=A_{k} \mathcal{R}_{[k, N ; N]} A_{k}^{T}+\sum_{l=1}^{m} \Pi_{l} \operatorname{tr}\left(\mathcal{P}_{[k, k ; N]} \Gamma_{l}\right)+D_{k} Q D_{k}^{T}+\Delta_{k+1}^{\mathcal{H}} \\
& \Upsilon_{k+1}=\Xi C_{k+1}\left(\overline{\mathcal{R}}_{[k, N ; N]}+\Delta_{k+1}^{\mathcal{H}}\right) C_{k+1}^{T} \Xi+\mathcal{S}_{[k+1 ; N+1]} .
\end{aligned}
$$


Furthermore, the relationship between $H_{[k+1, i ; N+1]}$ and $H_{[k, i ; N]}$ can be described by

$$
\left\{\begin{array}{l}
H_{[k+1, k+1 ; N+1]}=K_{k+1}, \\
H_{[k+1, i ; N+1]}=\left(A_{k}-K_{k+1} \Xi C_{k+1} A_{k}\right) H_{[k, i ; N]}, \quad k_{N} \leq i \leq k .
\end{array}\right.
$$

Proof. First, (9) can be rewritten as

$$
\begin{aligned}
\hat{x}_{(k+1 \mid k+1 ; N+1)} & =\left(A_{k}-K_{k+1} \Xi C_{k+1} A_{k}\right) \hat{x}_{(k \mid k ; N)}+K_{k+1} y_{k+1} \\
& =\sum_{i=k_{N}}^{k}\left(A_{k}-K_{k+1} \Xi C_{k+1} A_{k}\right) H_{[k, i ; N]} y_{i}+K_{k+1} y_{k+1} .
\end{aligned}
$$

Then, for $k_{N} \leq i \leq k$, it is not difficult to see that

$$
\begin{aligned}
& \mathrm{E}\left\{\left(x_{k+1}-\hat{x}_{(k+1 \mid k+1 ; N+1)}\right) y_{i}^{T} \mid \mathcal{I}_{k+1}^{N+1}\right\} \\
= & \mathrm{E}\left\{\left(x_{k+1}-A_{k} \hat{x}_{(k \mid k ; N)}\right) y_{i}^{T} \mid \mathcal{I}_{k+1}^{N+1}\right\} \\
& -K_{k+1} \mathrm{E}\left\{\left(y_{k+1}-\Xi C_{k+1} A_{k} \hat{x}_{(k \mid k ; N)}\right) y_{i}^{T} \mid \mathcal{I}_{k+1}^{N+1}\right\} \\
= & \mathrm{E}\left\{A_{k}\left(x_{k}-\hat{x}_{(k \mid k ; N)}\right) y_{i}^{T} \mid \mathcal{I}_{k+1}^{N+1}\right\}+\mathrm{E}\left\{\left(f\left(x_{k}, \vartheta_{k}\right)+D_{k} w_{k}\right) y_{i}^{T} \mid \mathcal{I}_{k+1}^{N+1}\right\} \\
& -K_{k+1} \mathrm{E}\left\{\Xi C_{k+1} A_{k}\left(x_{k}-\hat{x}_{(k \mid k ; N)}\right) y_{i}^{T} \mid \mathcal{I}_{k+1}^{N+1}\right\} \\
& -K_{k+1} \mathrm{E}\left\{\left(\left(\Xi_{k+1}-\Xi\right) C_{k+1} A_{k} x_{k}\right.\right. \\
& \left.\left.+\Xi_{k+1} C_{k+1}\left(f\left(x_{k}, \vartheta_{k}\right)+D_{k} w_{k}\right)+E_{k+1} v_{k+1}\right) y_{i}^{T} \mid \mathcal{I}_{k+1}^{N+1}\right\} \\
= & 0 .
\end{aligned}
$$

Similarly, for $i=k+1$, one has

$$
\begin{aligned}
& \mathrm{E}\left\{\left(x_{k+1}-\hat{x}_{(k+1 \mid k+1 ; N)}\right) y_{k+1}^{T} \mid \mathcal{I}_{k+1}^{N+1}\right\} \\
= & \mathrm{E}\left\{\left(x_{k+1}-A_{k} \hat{x}_{(k \mid k ; N)}\right) y_{k+1}^{T}-K_{k+1} \Xi C_{k+1}\left(x_{k+1}-A_{k} \hat{x}_{(k \mid k ; N)}\right) y_{k+1}^{T}\right. \\
& \left.-K_{k+1}\left(\left(\Xi_{k+1}-\Xi\right) C_{k+1} x_{k+1}+E_{k+1} v_{k+1}\right) y_{k+1}^{T} \mid \mathcal{I}_{k+1}^{N+1}\right\} \\
= & \left(I-K_{k+1} \Xi C_{k+1}\right) \mathrm{E}\left\{\left(x_{k+1}-A_{k} \hat{x}_{(k \mid k ; N)}\right) y_{k+1}^{T} \mid \mathcal{I}_{k+1}^{N+1}\right\}-K_{k+1} \mathcal{S}_{[k+1 ; N+1]}
\end{aligned}
$$

On the other hand, similar to Kwon et al. (1989), over the interval $k_{N} \leq i \leq$ $k_{N}+n$, one has

$$
\mathcal{R}_{[k, n ; N]}:=\mathcal{P}_{\left[k_{N}+n, k_{N}+n ; N\right]}-\sum_{s=k_{N}}^{k_{N}+n} H_{[k, s ; N]} \Xi C_{s} \mathcal{P}_{\left[s, k_{N}+n ; N\right]}
$$


Then, it follows from (14) and (15) that

$$
\begin{aligned}
& \mathrm{E}\left\{\left(x_{k+1}-A_{k} \hat{x}_{(k \mid k ; N)}\right) y_{k+1}^{T} \mid \mathcal{I}_{k+1}^{N+1}\right\} \\
= & \mathrm{E}\left\{x_{k+1} x_{k+1}^{T} C_{k+1}^{T} \Xi_{k+1} \mid \mathcal{I}_{k+1}^{N+1}\right\}-\sum_{s=k_{N}}^{k} A_{k} H_{[k, s ; N]} \Xi C_{s} \mathrm{E}\left\{x_{s} x_{k+1}^{T} \mid \mathcal{I}_{k+1}^{N+1}\right\} C_{k+1}^{T} \Xi \\
= & \left(\mathcal{P}_{[k+1, k+1 ; N+1]}-A_{k} \sum_{s=k_{N}}^{k} H_{[k, s ; N]} \Xi C_{s} \mathcal{P}_{[s, k ; N+1]} A_{k}^{T}\right) C_{k+1}^{T} \Xi \\
= & \left(\mathcal{P}_{[k+1, k+1 ; N+1]}+A_{k}\left(\mathcal{R}_{[k, N ; N]}-\mathcal{P}_{[k, k ; N]}\right) A_{k}^{T}\right) C_{k+1}^{T} \Xi \\
& -A_{k} \sum_{s=k_{N}}^{k} H_{[k, s ; N]} \Xi C_{s} \Delta_{[s, k]}^{\mathcal{P}} A_{k}^{T} C_{k+1}^{T} \Xi \\
= & \left(A_{k} \mathcal{R}_{[k, N ; N]} A_{k}^{T}+\sum_{l=1}^{m} \Pi_{l} \operatorname{tr}\left(\mathcal{P}_{[k, k ; N]} \Gamma_{l}\right)+D_{k} Q D_{k}^{T}\right) C_{k+1}^{T} \Xi \\
& +\left(\Delta_{[k+1, k+1]}^{\mathcal{P}}-A_{k} \sum_{s=k_{N}}^{k} H_{[k, s ; N]} \Xi C_{s} \Delta_{[s, k+1]}^{\mathcal{P}}\right) C_{k+1}^{T} \Xi \\
= & \left(\overline{\mathcal{R}}_{[k, N ; N]}+\Delta_{k+1}^{\mathcal{H}}\right) C_{k+1}^{T} \Xi .
\end{aligned}
$$

Therefore, in light of the orthogonality, substituting (16) into (14) results in (10).

Finally, we can calculate the filtering error covariance:

$$
\begin{aligned}
& \mathcal{R}_{[k+1, N+1 ; N+1]} \\
= & \mathrm{E}\left\{\left(x_{k+1}-\hat{x}_{(k+1 \mid k+1 ; N+1)}\right)\left(x_{k+1}-\hat{x}_{(k+1 \mid k+1 ; N+1)}\right)^{T} \mid \mathcal{I}_{k+1}^{N+1}\right\} \\
= & \frac{1}{2} \mathrm{E}\left\{\left(x_{k+1}-\hat{x}_{(k+1 \mid k+1 ; N+1)}\right) x_{k+1}^{T}+x_{k+1}\left(x_{k+1}-\hat{x}_{(k+1 \mid k+1 ; N+1)}\right)^{T} \mid \mathcal{I}_{k+1}^{N+1}\right\} \\
= & \frac{1}{2} \mathrm{E}\left\{\left[\left(I-K_{k+1} \Xi C_{k+1}\right) A_{k}\left(x_{k}-\hat{x}_{(k \mid k ; N)}\right)+\left(I-K_{k+1} \Xi_{k+1} C_{k+1}\right)\left(f\left(x_{k}, \vartheta_{k}\right)+D_{k} w_{k}\right)\right.\right. \\
& \left.-K_{k+1}\left(\Xi_{k+1}-\Xi\right) C_{k+1} A_{k} x_{k}-K_{k+1} E_{k+1} v_{k+1}\right]\left[A_{k} x_{k}+f\left(x_{k}, \vartheta_{k}\right)+D_{k} w_{k}\right]^{T} \\
& +\left[A_{k} x_{k}+f\left(x_{k}, \vartheta_{k}\right)+D_{k} w_{k}\right]\left[\left(I-K_{k+1} \Xi C_{k+1}\right) A_{k}\left(x_{k}-\hat{x}_{(k \mid k ; N)}\right)\right. \\
& +\left(I-K_{k+1} \Xi_{k+1} C_{k+1}\right)\left(f\left(x_{k}, \vartheta_{k}\right)+D_{k} w_{k}\right) \\
& \left.\left.-K_{k+1}\left(\Xi_{k+1}-\Xi\right) C_{k+1} A_{k} x_{k}-K_{k+1} E_{k+1} v_{k+1}\right]^{T} \mid \mathcal{I}_{k+1}^{N+1}\right\} \\
= & \frac{1}{2}\left\{\left(I-K_{k+1} \Xi C_{k+1}\right) \Delta_{\mathcal{R}}^{\mathcal{H}}+\left(\Delta_{\mathcal{R}}^{\mathcal{H}}\right)^{T}\left(I-K_{k+1} \Xi C_{k+1}\right)^{T}\right\},
\end{aligned}
$$

which completes the proof. 


\subsection{The estimation of state covariance matrix}

As discussed in Introduction, the state covariance matrix $\mathcal{P}_{\left[k_{N}-1, k_{N}-1 ; N\right]}$ has to be estimated by means of the known measurement set $\mathcal{I}_{k}^{N+1}$. Obtaining such an estimation can be divided into two steps, that is, 1) obtaining the unbiased estimate $\hat{x}_{\left(k_{N}-1 \mid k\right)}$ and 2) calculating the state covariance matrix. Now, let us introduce the following assumption. It should be pointed out that, for the case of linear timeinvariant systems, such an assumption is equivalent to the observability condition which is a basic requirement for state estimation problems.

Assumption 1. For all $k \geq 0, r\left(E_{k} R E_{k}^{T}\right)=n_{y}$ and $r\left(\chi_{k}\right)=n_{x}$, where

$$
\chi_{k}:=\left[\begin{array}{llll}
C_{k_{n}-1}^{T} & A_{k-1}^{T} C_{k_{n}}^{T} \quad \cdots \quad\left(A_{k-1} A_{k-2} \cdots A_{k_{n}+n-1}\right)^{T} C_{k}^{T}
\end{array}\right]^{T}, \quad n \geq n_{x} .
$$

Firstly, let us obtain the unbiased estimate $\hat{x}_{\left(k_{N}-1 \mid k\right)}$ of the initial state $x_{k_{N}-1}$ on each sliding-window. For this purpose, denoting

$$
\begin{aligned}
Y_{k} & =\left[y_{k}^{T}, y_{k-1}^{T}, \cdots, y_{k_{N}-1}^{T}\right]^{T}, \quad \tilde{w}_{k}=\left[w_{k-1}^{T}, w_{k-2}^{T}, \cdots, w_{k_{N}-1}^{T}\right]^{T}, \\
\tilde{v}_{k} & =\left[v_{k}^{T}, v_{k-1}^{T}, \cdots, v_{k_{N}-1}^{T}\right]^{T}, \quad \Theta_{k}=\operatorname{diag}\left\{\Xi_{k}, \Xi_{k-1}, \cdots, \Xi_{k_{N}-1}\right\}, \\
\tilde{f}_{k} & =\left[f^{T}\left(x_{k-1}, \vartheta_{k-1}\right) f^{T}\left(x_{k-2}, \vartheta_{k-2}\right) \cdots f^{T}\left(x_{k_{N}-1}, \vartheta_{k_{N}-1}\right)\right]^{T},
\end{aligned}
$$

one has

$$
Y_{k}=\Theta_{k} \mathcal{C}_{k} x_{k_{N}-1}+\Theta_{k} \mathcal{F}_{k} \tilde{f}_{k}+\Theta_{k} \mathcal{D}_{k} \tilde{w}_{k}+\mathcal{E}_{k} \tilde{v}_{k}
$$

where

$$
\begin{aligned}
& \mathcal{C}_{k}=\left[\mathcal{A}_{\left(k-1: k_{N}-1\right)}^{T} C_{k}^{T}, \mathcal{A}_{\left(k-2: k_{N}-1\right)}^{T} C_{k-1}^{T}, \cdots \mathcal{A}_{\left(k_{N}-2: k_{N}-1\right)}^{T} C_{k_{N}-1}^{T}\right]^{T}, \\
& \mathcal{E}_{k}=\operatorname{diag}\left\{E_{k}, E_{k-1}, \cdots, E_{k_{N}}, E_{k_{N}-1}\right\}, \quad \overline{\mathcal{C}}_{(m: n)}=C_{m} \mathcal{A}_{(m-1: n)}, \\
& \mathcal{D}_{k}=\mathcal{F}_{k} \overline{\mathcal{D}}_{k}, \quad \overline{\mathcal{D}}_{k}=\operatorname{diag}\left\{D_{k-1}, D_{k-2}, \cdots, D_{k_{N}-1}\right\} \text {, } \\
& \mathcal{F}_{k}=\left[\begin{array}{ccccc}
\overline{\mathcal{C}}_{(k: k)} & \overline{\mathcal{C}}_{(k: k-1)} & \cdots & \overline{\mathcal{C}}_{\left(k: k_{N}+1\right)} & \overline{\mathcal{C}}_{\left(k: k_{N}\right)} \\
0 & \overline{\mathcal{C}}_{(k-1: k-1)} & \cdots & \overline{\mathcal{C}}_{\left(k-1: k_{N}+1\right)} & \overline{\mathcal{C}}_{\left(k-1: k_{N}\right)} \\
\vdots & \ddots & \vdots & \vdots & \vdots \\
0 & 0 & \cdots & \overline{\mathcal{C}}_{\left(k_{N}+1: k_{N}+1\right)} & \overline{\mathcal{C}}_{\left(k_{N}+1: k_{N}\right)} \\
0 & 0 & \cdots & 0 & \overline{\mathcal{C}}_{\left(k_{N}: k_{N}\right)} \\
0 & 0 & \cdots & 0 & 0
\end{array}\right]
\end{aligned}
$$

Define $\hbar_{k}:=\Theta_{k} \mathcal{C}_{k} x_{k_{N}-1}+\Theta_{k} \mathcal{F}_{k} \tilde{f}_{k}+\Theta_{k} \mathcal{D}_{k} \tilde{w}_{k}+\mathcal{E}_{k} \tilde{v}_{k}-Y_{k}$ and $\bar{\Theta}=I \otimes \Xi$. It follows that

$$
\begin{aligned}
& \mathrm{E}\left\{\hbar_{k}^{T} \hbar_{k} \mid x_{k_{N}-1}\right\} \\
= & \mathrm{E}\left\{x_{k_{N}-1}^{T} \mathcal{C}_{k}^{T} \Theta_{k}^{T} \Theta_{k} \mathcal{C}_{k} x_{k_{N}-1}+2 x_{k_{N}-1}^{T} \mathcal{C}_{k}^{T} \Theta_{k}^{T}\left(\Theta_{k} \mathcal{F}_{k} \tilde{f}_{k}+\Theta_{k} \mathcal{D}_{k} \tilde{w}_{k}+\mathcal{E}_{k} \tilde{v}_{k}\right)\right. \\
& -2 x_{k_{N}-1}^{T} \mathcal{C}_{k}^{T} \Theta_{k}^{T} Y_{k}+\tilde{f}_{k}^{T} \mathcal{F}_{k}^{T} \Theta_{k}^{T} \Theta_{k} \mathcal{F}_{k} \tilde{f}_{k}+2 \tilde{f}_{k}^{T} \mathcal{F}_{k}^{T} \Theta_{k}^{T}\left(\Theta_{k} \mathcal{D}_{k} \tilde{w}_{k}+\mathcal{E}_{k} \tilde{v}_{k}-Y_{k}\right) \\
& \left.+\left(\Theta_{k} \mathcal{D}_{k} \tilde{w}_{k}+\mathcal{E}_{k} \tilde{v}_{k}\right)^{T}\left(\Theta_{k} \mathcal{D}_{k} \tilde{w}_{k}+\mathcal{E}_{k} \tilde{v}_{k}\right)-2\left(\Theta_{k} \mathcal{D}_{k} \tilde{w}_{k}+\mathcal{E}_{k} \tilde{v}_{k}\right)^{T} Y_{k}+Y_{k}^{T} Y_{k} \mid x_{k_{N}-1}\right\}
\end{aligned}
$$


which results in

$$
\begin{aligned}
& \mathrm{E}\left\{\hbar_{k}^{T} \hbar_{k} \mid x_{k_{N}-1}\right\} \\
= & x_{k_{N}-1}^{T} \mathcal{C}_{k}^{T} \bar{\Theta} \mathcal{C}_{k} x_{k_{N}-1}-2 x_{k_{N}-1}^{T} \mathcal{C}_{k}^{T} \bar{\Theta} Y_{k}+\mathrm{E}\left\{\tilde{f}_{k}^{T} \mathcal{F}_{k}^{T} \bar{\Theta} \mathcal{F}_{k} \tilde{f}_{k} \mid x_{k_{N}-1}\right\} \\
& +\mathrm{E}\left\{\left(\Theta_{k} \mathcal{D}_{k} \tilde{w}_{k}+\mathcal{E}_{k} \tilde{v}_{k}\right)^{T}\left(\Theta_{k} \mathcal{D}_{k} \tilde{w}_{k}+\mathcal{E}_{k} \tilde{v}_{k}\right)\right\}+Y_{k}^{T} Y_{k} .
\end{aligned}
$$

Furthermore, denote

$$
\begin{aligned}
\varphi_{k}:= & x_{k_{N}-1}^{T} \mathcal{C}_{k}^{T} \bar{\Theta} \mathcal{C}_{k} x_{k_{N}-1}-2 x_{k_{N}-1}^{T} \mathcal{C}_{k}^{T} \bar{\Theta} Y_{k} \\
& +\mathrm{E}\left\{\left(\Theta_{k} \mathcal{D}_{k} \tilde{w}_{k}+\mathcal{E}_{k} \tilde{v}_{k}\right)^{T}\left(\Theta_{k} \mathcal{D}_{k} \tilde{w}_{k}+\mathcal{E}_{k} \tilde{v}_{k}\right)\right\}+Y_{k}^{T} Y_{k}
\end{aligned}
$$

and setting the derivative $d \varphi_{k} / d x_{k_{N}-1}$ to zero yields

$$
\tilde{x}_{\left(k_{N}-1 \mid k\right)}:=\left(\mathcal{C}_{k}^{T} \bar{\Theta} \mathcal{C}_{k}\right)^{-1} \mathcal{C}_{k}^{T} \bar{\Theta} Y_{k}=\mathcal{N}_{k} Y_{k}
$$

with $\mathcal{N}_{k}:=\left(\mathcal{C}_{k}^{T} \bar{\Theta} \mathcal{C}_{k}\right)^{-1} \mathcal{C}_{k}^{T} \bar{\Theta}$.

On the other hand, taking the above equation into consideration, one has

$$
\begin{aligned}
& \mathrm{E}\left\{x_{k_{N}-1}-\tilde{x}_{\left(k_{N}-1 \mid k\right)} \mid \mathcal{I}_{k}^{N+1}\right\} \\
= & \mathrm{E}\left\{x_{k_{N}-1}-\mathcal{N}_{k} Y_{k} \mid \mathcal{I}_{k}^{N+1}\right\} \\
= & \mathrm{E}\left\{x_{k_{N}-1}-\mathcal{N}_{k}\left(\Theta_{k} \mathcal{C}_{k} x_{k_{N}-1}+\Theta_{k} \mathcal{F}_{k} \tilde{f}_{k}+\Theta_{k} \mathcal{D}_{k} \tilde{w}_{k}+\mathcal{E}_{k} \tilde{v}_{k}\right) \mid \mathcal{I}_{k}^{N+1}\right\} \\
= & \mathrm{E}\left\{x_{k_{N}-1}-\mathcal{N}_{k} \Theta_{k} \mathcal{C}_{k} x_{k_{N}-1} \mid \mathcal{I}_{k}^{N+1}\right\} \\
= & \mathrm{E}\left\{x_{k_{N}-1}-\mathcal{N}_{k} \bar{\Theta} \mathcal{C}_{k} x_{k_{N}-1}-\mathcal{N}_{k}\left(\Theta_{k}-I\right) \mathcal{C}_{k} x_{k_{N}-1} \mid \mathcal{I}_{k}^{N+1}\right\} \\
= & \mathrm{E}\left\{\left(I-\mathcal{N}_{k} \bar{\Theta} \mathcal{C}_{k}\right) x_{k_{N}-1} \mid \mathcal{I}_{k}^{N+1}\right\} .
\end{aligned}
$$

which means that the unbiased estimate $\hat{x}_{\left(k_{N}-1 \mid k\right)}$ is

$$
\mathcal{N}_{k} \bar{\Theta} \mathcal{C}_{k} \hat{x}_{\left(k_{N}-1 \mid k\right)}=\tilde{x}_{\left(k_{N}-1 \mid k\right)}, \quad \text { or } \quad \hat{x}_{\left(k_{N}-1 \mid k\right)}=\left(\mathcal{C}_{k}^{T} \bar{\Theta}^{2} \mathcal{C}_{k}\right)^{-1} \mathcal{C}_{k}^{T} \bar{\Theta} Y_{k}
$$

Now, we are in a position to obtain the estimate $\hat{\mathcal{P}}_{\left[k_{N}-1, k_{N}-1 ; N\right]}$ of the state covariance matrix $\mathcal{P}_{\left[k_{N}-1, k_{N}-1 ; N\right]}$. It is easily found that

$$
\begin{aligned}
& \mathrm{E}\left\{\left(\mathcal{N}_{k} \bar{\Theta} \mathcal{C}_{k} x_{k_{N}-1}-\tilde{x}_{\left(k_{N}-1 \mid k\right)}\right)\left(\mathcal{N}_{k} \bar{\Theta} \mathcal{C}_{k} x_{k_{N}-1}-\tilde{x}_{\left(k_{N}-1 \mid k\right)}\right)^{T} \mid \mathcal{I}_{k}^{N+1}\right\} \\
= & \mathrm{E}\left\{\left(\mathcal{N}_{k}\left(\bar{\Theta}-\Theta_{k}\right) \mathcal{C}_{k} x_{k_{N}-1}-\mathcal{N}_{k}\left(\Theta_{k} \mathcal{F}_{k} \tilde{f}_{k}+\Theta_{k} \mathcal{D}_{k} \tilde{w}_{k}+\mathcal{E}_{k} \tilde{v}_{k}\right)\right)\right. \\
& \left.\times\left(\mathcal{N}_{k}\left(\bar{\Theta}-\Theta_{k}\right) \mathcal{C}_{k} x_{k_{N}-1}-\mathcal{N}_{k}\left(\Theta_{k} \mathcal{F}_{k} \tilde{f}_{k}+\Theta_{k} \mathcal{D}_{k} \tilde{w}_{k}+\mathcal{E}_{k} \tilde{v}_{k}\right)\right)^{T} \mid \mathcal{I}_{k}^{N+1}\right\} \\
= & \mathcal{N}_{k}\left(\mathcal{E}_{k}(I \otimes R) \mathcal{E}_{k}^{T}+\tilde{\Theta} \circ\left(\mathcal{C}_{k} \mathcal{P}_{\left[k_{N}-1, k_{N}-1 ; N\right]} \mathcal{C}_{k}^{T}\right)+\bar{\Theta} \mathcal{F}_{k} \mathrm{E}\left\{\tilde{f}_{k} \tilde{f}_{k}^{T}\right\} \mathcal{F}_{k}^{T} \bar{\Theta}\right. \\
& \left.+\tilde{\Theta} \circ\left(\mathcal{F}_{k} \mathrm{E}\left\{\tilde{f}_{k} \tilde{f}_{k}^{T}\right\} \mathcal{F}_{k}^{T}\right)+\bar{\Theta} \mathcal{D}_{k}(I \otimes Q) \mathcal{D}_{k}^{T} \bar{\Theta}+\tilde{\Theta} \circ\left(\mathcal{D}_{k}(I \otimes Q) \mathcal{D}_{k}^{T}\right)\right) \mathcal{N}_{k}^{T} \\
= & \mathcal{N}_{k}\left(\mathcal{E}_{k}(I \otimes R) \mathcal{E}_{k}^{T}+\tilde{\Theta} \circ\left(\mathcal{C}_{k} \mathcal{P}_{\left[k_{N}-1, k_{N}-1 ; N\right]} \mathcal{C}_{k}^{T}\right)+\bar{\Theta} \mathcal{F}_{k} \Omega_{f}(\mathcal{P}) \mathcal{F}_{k}^{T} \bar{\Theta}\right. \\
& \left.+\tilde{\Theta} \circ\left(\mathcal{F}_{k} \Omega_{f}(\mathcal{P}) \mathcal{F}_{k}^{T}\right)+\bar{\Theta} \mathcal{D}_{k}(I \otimes Q) \mathcal{D}_{k}^{T} \bar{\Theta}+\tilde{\Theta} \circ\left(\mathcal{D}_{k}(I \otimes Q) \mathcal{D}_{k}^{T}\right)\right) \mathcal{N}_{k}^{T}
\end{aligned}
$$


where $\tilde{\Theta}=I \otimes \tilde{\Xi}$ and

$\Omega_{f}(\mathcal{P}):=\mathrm{E}\left\{\tilde{f}_{k} \tilde{f}_{k}^{T}\right\}=\sum_{l}^{m}\left(I \otimes \Pi_{l}\right) \operatorname{diag}\left\{\operatorname{tr}\left(\mathcal{P}_{[k-1, k-1 ; N]} \Gamma_{l}\right), \cdots, \operatorname{tr}\left(\mathcal{P}_{\left[k_{N}-1, k_{N}-1 ; N\right]} \Gamma_{l}\right)\right\}$

Considering $\mathrm{E}\left\{\mathcal{N}_{k} \bar{\Theta} \mathcal{C}_{k} x_{k_{N}-1} \mid \mathcal{I}_{k}^{N+1}\right\}=\mathcal{N}_{k} Y_{k}$, one has

$$
\begin{aligned}
\mathrm{E}\left\{\left(\mathcal{N}_{k} \bar{\Theta} \mathcal{C}_{k} x_{k_{N}-1}-\tilde{x}_{\left(k_{N}-1 \mid k\right)}\right)\right. & \left.\left(\mathcal{N}_{k} \bar{\Theta} \mathcal{C}_{k} x_{k_{N}-1}-\tilde{x}_{\left(k_{N}-1 \mid k\right)}\right)^{T} \mid \mathcal{I}_{k}^{N+1}\right\} \\
= & \mathcal{N}_{k} \bar{\Theta} \mathcal{C}_{k} \mathcal{P}_{\left[k_{N}-1, k_{N}-1 ; N\right]} \mathcal{C}_{k}^{T} \bar{\Theta} \mathcal{N}_{k}^{T}-\mathcal{N}_{k} Y_{k} Y_{k}^{T} \mathcal{N}_{k}^{T}
\end{aligned}
$$

Therefore, according to (21) and (22), we have the following theorem.

Theorem 2. For the given information set $\mathcal{I}_{k}^{N+1}$, the estimate $\hat{\mathcal{P}}_{\left[k_{N}-1, k_{N}-1 ; N\right]}$ of the state covariance matrix $\mathcal{P}_{\left[k_{N}-1, k_{N}-1 ; N\right]}$ satisfies the following nonlinear matrix equation:

$$
\hat{\mathcal{P}}_{\left[k_{N}-1, k_{N}-1 ; N\right]}=\left(\mathcal{C}_{k}^{T} \bar{\Theta}^{2} \mathcal{C}_{k}\right)^{-1}(\mathcal{L}(\hat{\mathcal{P}})+\mathcal{J}(Y))\left(\mathcal{C}_{k}^{T} \bar{\Theta}^{2} \mathcal{C}_{k}\right)^{-1}
$$

with

$$
\left\{\begin{aligned}
\mathcal{L}(\hat{\mathcal{P}}):= & \mathcal{C}_{k}^{T} \bar{\Theta}\left(\tilde{\Theta} \circ\left(\mathcal{F}_{k} \Omega_{f}(\hat{\mathcal{P}}) \mathcal{F}_{k}^{T}\right)\right. \\
& \left.+\tilde{\Theta} \circ\left(\mathcal{C}_{k} \hat{\mathcal{P}}_{\left[k_{N}-1, k_{N}-1 ; N\right]} \mathcal{C}_{k}^{T}\right)+\bar{\Theta} \mathcal{F}_{k} \Omega_{f}(\hat{\mathcal{P}}) \mathcal{F}_{k}^{T} \bar{\Theta}\right) \bar{\Theta} \mathcal{C}_{k} \\
\mathcal{J}(Y):= & \mathcal{C}_{k}^{T} \bar{\Theta}\left(\mathcal{E}_{k}(I \otimes R) \mathcal{E}_{k}^{T}+\bar{\Theta} \mathcal{D}_{k}(I \otimes Q) \mathcal{D}_{k}^{T} \bar{\Theta}\right. \\
& \left.+\tilde{\Theta} \circ\left(\mathcal{D}_{k}(I \otimes Q) \mathcal{D}_{k}^{T}\right)+Y_{k} Y_{k}^{T}\right) \bar{\Theta} \mathcal{C}_{k}
\end{aligned}\right.
$$

Remark 1. Note that the nonlinear matrix equation (23) is solvable if there exists a scalar $\lambda>0$ satisfying $\left(\mathcal{C}_{k}^{T} \bar{\Theta}^{2} \mathcal{C}_{k}\right)^{2}-\lambda^{-1}(\mathcal{L}(\lambda I)+\mathcal{N}(Y))>0$. Furthermore, its approximate solution can be acquired by using some basic iterative methods, such as Aitken's multi-point iterative algorithm, Newton iterative method, and so forth.

Remark 2. In most existing literature, three arguably theoretical frameworks, namely, the setting values, the least-squares technique and the maximum-likelihood method, have been widely exploited to estimate the covariance matrix. Unfortunately, all of these methods are no longer valid because of the nonlinear operation (i.e. the "trace" and Hadamard-product operations) included in (21) and the unknown probability density function of the augmented measurement $Y_{k}$ with missing measurements. As such, in this paper, a novel two-stage scheme is provided to overcome such a challenge. It is worth mentioning that the state covariance $\mathcal{R}_{[k, N ; N]}$ are affected by both missing measurements and finite measurement data. An important research topic in future is how to reduce such an effect from these two aspects.

\section{A Numerical Example}

In this section, a numerical example is utilized to illustrate the effectiveness of the established filtering algorithm for discrete time-varying systems (1) with missing 
measurements. Consider the system with

$$
\begin{gathered}
A_{k}=\left[\begin{array}{ccc}
0.64+0.22 \sin (0.12 k) & 0.40 & 0 \\
0.25 & 0.32+0.10 \cos (2 k) & 0.10 \\
0.20 & -0.30 & 0.45
\end{array}\right], \\
C_{k}=\left[\begin{array}{cc}
0.82 & 0.15 \\
0.75 & 0 \\
0 & -0.80
\end{array}\right]^{T}, D_{k}=\left[\begin{array}{c}
0.10 \\
0 \\
-0.05
\end{array}\right], E_{k}=\left[\begin{array}{cc}
-0.05 & 0.20 \\
0.10 & -0.15
\end{array}\right] .
\end{gathered}
$$

Then, the nonlinear vector-valued function $f_{k}\left(x_{k}, \vartheta_{k}\right)$ is chosen as

$$
\left.f_{k}\left(x_{k}, \vartheta_{k}\right)=\left(0.1 \operatorname{sign}\left(x_{k}^{1}\right) x_{k}^{1} \vartheta_{k}^{1}+0.2 \operatorname{sign}\left(x_{k}^{2}\right) x_{k}^{2} \vartheta_{k}^{2}\right)\right)\left[\begin{array}{c}
0.06 \\
0.09 \\
0.12
\end{array}\right]
$$

where $x_{k}^{i}$ and $\vartheta_{k}^{i}$ are, respectively, the $i$-th element of the system state $x_{k}$ and the stochastic variable $\vartheta_{k}$. Furthermore, $\vartheta_{k}^{1}$ and $\vartheta_{k}^{2}$ are zero mean, uncorrelated Gaussian white noise sequences with unity covariances. The probabilities of multiple missing measurements are taken as $\bar{\alpha}_{1}=0.98$ and $\bar{\alpha}_{2}=0.96$. The $N$ is selected as 3 . It is not difficult to verify that the above stochastic nonlinear function satisfies

$$
\begin{aligned}
& \mathrm{E}\left\{f\left(x_{k}, \vartheta_{k}\right) \mid x_{k}\right\}=0, \\
& \mathrm{E}\left\{f\left(x_{k}, \vartheta_{k}\right) f^{T}\left(x_{k}, \vartheta_{k}\right) \mid x_{k}\right\}=\left[\begin{array}{l}
0.06 \\
0.09 \\
0.12
\end{array}\right]\left[\begin{array}{l}
0.06 \\
0.09 \\
0.12
\end{array}\right]^{T} x_{k}^{T}\left[\begin{array}{ccc}
0.01 & 0 & 0 \\
0 & 0.04 & 0 \\
0 & 0 & 0
\end{array}\right] x_{k} .
\end{aligned}
$$

The simulation results are shown in Fig. 1, where Figs. 1(a) 1(c) plot the system state and its estimation, and Figs. 1(d) 1(f) depict the absolute values of filtering errors for the addressed system. The simulation results have confirmed that the designed filter performance is very well.

\section{Conclusions}

In this paper, we have investigated the receding horizon filtering problem for a class of discrete time-varying nonlinear systems. In order to reflect the reality more closely, on one hand, the phenomenon of multiple missing measurements is addressed, where the missing probability of each sensor is different. On the other hand, the stochastic nonlinearity which covers several classes of well-studied nonlinearities as special cases, has been taken into account. In terms of the projection theory combined with stochastic analysis techniques, a Kalman-type receding horizon filter has been designed. Furthermore, by utilizing the conditional expectation, a novel estimation scheme has been proposed to obtain the estimation of the state covariance matrix. Further research topics include the extension of our results to more general networked systems with various network-induced phenomena, such as stochastic occurring nonlinearities, time-delays, quantization effect and so forth. 


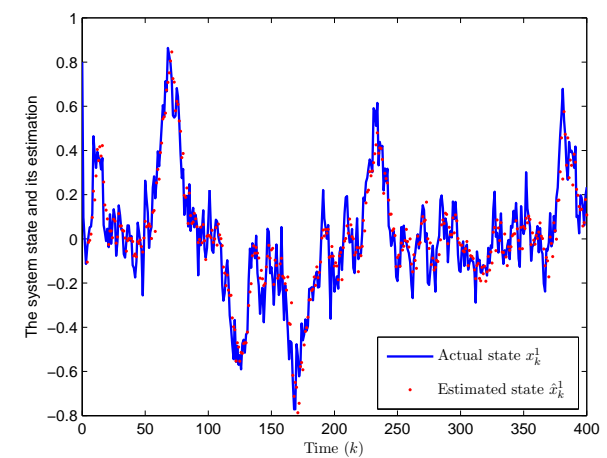

(a) The actual state $x_{1}^{k}$ and its estimation $\hat{x}_{1}^{k}$.

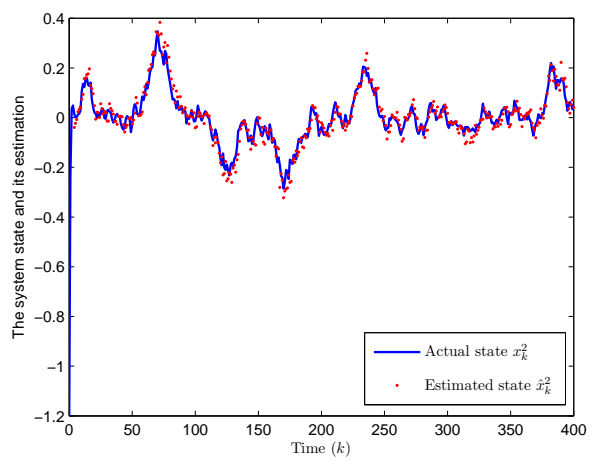

(b) The actual state $x_{2}^{k}$ and its estimation $\hat{x}_{2}^{k}$.

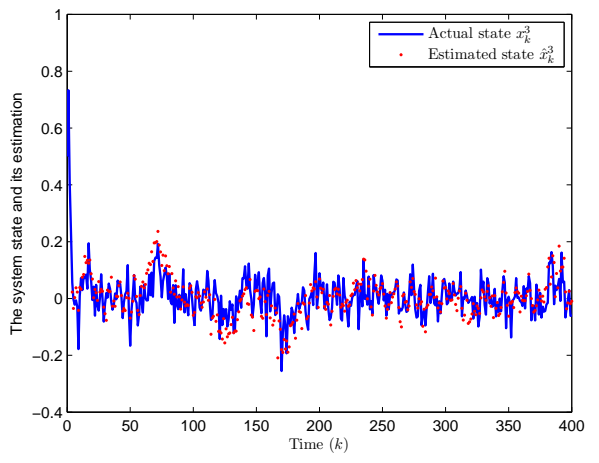

(c) The actual state $x_{3}^{k}$ and its estimation $\hat{x}_{3}^{k}$.

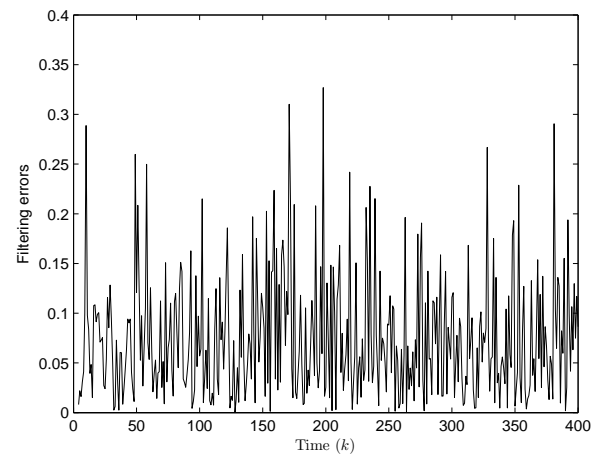

(d) Filtering errors of $x_{k}^{1}$.

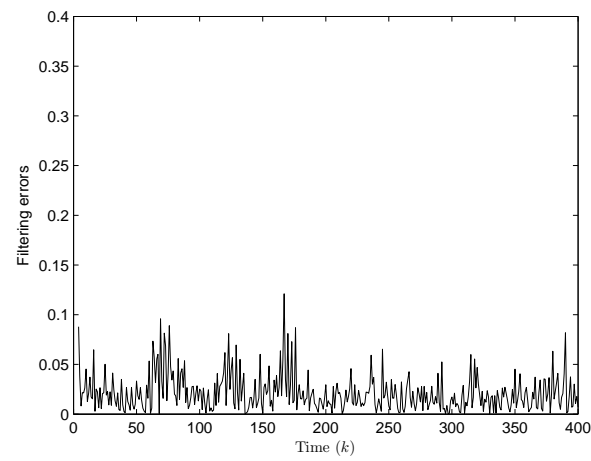

(e) Filtering errors of $x_{k}^{2}$.

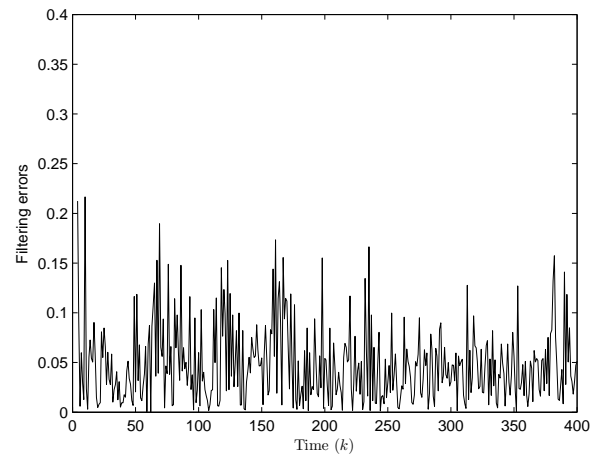

(f) Filtering errors of $x_{k}^{3}$.

Figure 1. The state estimation and filtering errors (absolute values).

\section{Funding}

This work was supported in part by the the Deanship of Scientific Research (DSR) at King Abdulaziz University in Saudi Arabia [grant number 16-135-35-HiCi], the National Natural Science Foundation of China [grant number 61329301], [grant number 61203139], [grant number 61134009], and [grant number 61104125], Royal Society of the U.K., the Shanghai Rising-Star Program of China grant number 13QA1400100], the Shu Guang project of Shanghai Municipal Education Commission and Shanghai Education Development Foundation [grant number 13SG34], the Fundamental Research Funds for the Central Universities, DHU Distinguished Young Professor Program, and the Alexander von Humboldt Foundation of Ger- 
many.

\section{References}

Alessandri, A., M. Baglietto, and G. Battistelli. 2005 Receding-horizon estimation for switching discrete-time linear systems, IEEE Transactions on Automatic Control, vol. 50, no. 11, pp. 1736-1748.

Alessandri, A., M. Baglietto, and G. Battistelli. 2003. Receding-horizon estimation for discrete-time linear systems, IEEE Transactions on Automatic Control, vol. 48, no. 3, pp. 473-478.

Bouibed, K., L. Seddiki, K. Guelton, and H. Akdag. 2014. Actuator and sensor fault detection and isolation of an actuated seat via nonlinear multi-observers, Systems Science and Control Engineering: An Open Access Journal, vol. 2, no. 1, pp. 150-160.

Buxbaum, P. 1974. Fixed-memory recursive filters, IEEE Transactions on Information Theory, vol. 20, no. 1, pp. 113-115.

Dong, H., Z. Wang, D. W. C. Ho, and H. Gao. 2010. Variance-constrained $H_{\infty}$ filtering for a class of nonlinear time-varying systems with multiple missing measurements: the finite-horizon case, IEEE Transactions on Signal Processing, vol. 58, no. 5, pp. 2534-2543.

Fitzgerald, R. J. 1971. Divergence of the Kalman filter, IEEE Transactions on Automatic Control, vol. 16, no. 6, pp. 736-747.

Friedlander, B., T. Kailath, and M. Morf. 1978. Extended Levinson and Chandrasekhar equations for general discrete-time linear estimation problems, IEEE Transactions on Automatic Control, vol. AC-23, no. 4, pp. 653-659.

Grewal, M. S., and A. P. Anderews. 1993. Kalman filtering: theory and practice, Englewood Cliffs, NJ:Prentice-Hall.

He, X., Z. Wang, X. Wang, and D. Zhou. 2014. Networked strong tracking filtering with multiple packet dropouts: algorithms and applications, IEEE Transactions on Industrial Electronics, vol. 61, no. 3, pp. 1454-1463.

Hu, J., Z. Wang, H. Gao, and L. K. Stergioulas. 2012. Extended Kalman filtering with stochastic nonlinearities and multiple missing measurements, Automatica, vol. 48 , no. 9 , pp. 2007-2015.

Hu, J., Z. Wang, B. Shen, and H. Gao. 2013. Quantised recursive filtering for a class of nonlinear systems with multiplicative noises and missing measurements, International Journal of Control, vol. 86, no. 4, pp. 650-663.

Hounkpevi, F. O., and E. Yaz. 2007. Robust minimum variance linear state estimators for multiple sensors with different failure rates, Automatica, vol. 43, no. 7, pp. 1274-1280.

Jacobson, D. 1974. A general result in stochastic optimal control of nonlinear discrete-time systems with quadratic performance criteria, Journal of Mathematical Analysis and Applications, vol. 47, no. 1, pp. 153-161.

Jazwinski, A. 1968. Limited memory optimal filtering, IEEE Transactions on Automatic Control, vol. 13, no. 5, pp. 558-563.

Kim, P. 2010. An alternative FIR filter for state estimation in discrete-time systems, Digital Signal Processing, vol. 20, no. 3, pp. 935-943.

Kluge, S., K. Reif, and M. Brokate. 2010. Stochastic stability of the extended Kalman filter with intermittent observations, IEEE Transactions on Automatic Control, vol. 55, no. 2, pp. 514-518, Feb. 2010.

Kwon, O., W. Kwon, and K. Lee. 1989. FIR filter and recursive forms for discretetime state-space models, Automatica, vol. 25, no. 5, pp. 715-728, May 1989. 
Kwon, W., K. Lee, and J. Lee. 1994. Fast algorithms for optimal FIR filter and smoother of discrete-time state-space models, Automatica, vol. 30, no. 3, pp. 489492, Mar. 1994.

Kwon, W., P. Kim, and S. Han. 2002. A receding horizon unbiased FIR filter for discrete-time state space models, Automatica, vol. 38, no. 3, pp. 545-551.

Liang, J., F. Sun, and X. Liu. 2014. Finite-horizon $H_{\infty}$ filtering for time-varying delay systems with randomly varying nonlinearities and sensor saturations, Systems Science and Control Engineering: An Open Access Journal, vol. 2, no. 1, pp. $108-118$.

Liu, L., F. Wu, and W. Zhang. 2014. Estimating parameters of S-systems by an auxiliary function guided coordinate descent method, Systems Science and Control Engineering: An Open Access Journal, vol. 2, no. 1, pp. 125-134.

Park, S., W. Kwon, O. Kwon, and M. Kim. 1997. Short-time Fourier analysis via optimal harmonic FIR filters, IEEE Transactions on Signal Processing, vol. 45, no. 6, pp. 1535-1542.

Sahebsara, M., T. Chen, and S. L. Shah. 2007. Optimal $H_{2}$ filtering in networked control systems with multiple packet dropout, IEEE Transactions on Automatic Control, vol. 52, no. 8, pp. 1508-1513.

Sangsuk-Iam, S., and T. E. Bullock. 1990. Analysis of discrete-time Kalman filtering under incorrect noise covariances, IEEE Transactions on Automatic Control, vol. 35, no. 5, pp. 1304.

Shmaliy, Y. S., J. Munoz-Diaz, and L. Arceo-Miquel. 2008. Optimal horizons for a one-parameter family of unbiased FIR filters, Digital Signal Processing, vol. 18, no. 5, pp. 739-750.

Shmaliy, Y. S. 2008. Optimal gains of FIR estimators for a class of discrete-time state-space models, IEEE Signal Processing Letters, vol. 15, pp. 517-520, 2008.

Shen, B., Z. Wang, and Y. S. Hung. 2010. Distributed $H_{\infty}$-consensus filtering in sensor networks with multiple missing measurements: the finite-horizon case, Automatica, vol. 46, no. 10, pp. 1682-1688.

Shen, B. 2014. A survey on the applications of the Krein-space theory in signal estimation, Systems Science and Control Engineering: An Open Access Journal, vol. 2, no. 1, pp. 143-149.

Sinopoli, B., L. Schenato, M. Franceschetti, K. Poolla, M. I. Jordan, and S. S. Sastry. 2004. Kalman filtering with intermittent observations, IEEE Transactions on Automatic Control, vol. 49, pp. 1453-1464.

Wang, Z., D. W. C. Ho, and X. Liu. 2003. Variance-constrained filtering for uncertain stochastic systems with missing measurements, IEEE Transactions on Automatic Control, vol. 48, no. 7, pp. 1254-1258.

Wei, G., Z. Wang, and H. Shu. 2009. Robust filtering with stochastic nonlinearities and multiple missing measurements, Automatica, vol. 45, no. 3, pp. 836-841.

Yaz, E., and Y. Yaz. 2001. State estimation of uncertain nonlinear stochastic systems with general criteria, Applied Mathematics Letters, vol. 14, no. 5, pp. 605610 . 